\title{
Propofol attenuates inflammatory damage on neurons following cerebral infarction by inhibiting excessive activation of microglia
}

\author{
HANG YU ${ }^{1}$, XIAOZHI WANG ${ }^{1}$, FUXIN KANG $^{1}$, ZHILE CHEN $^{1}$, YUNXIA MENG $^{1}$ and MINGMING DAI ${ }^{2}$ \\ ${ }^{1}$ Intensive Care Unit and ${ }^{2}$ Department of Internal Neurology, The Second Affiliated Hospital, \\ Hainan Medical University, Haikou, Hainan 570311, P.R. China
}

Received September 21, 2017; Accepted February 2, 2018

DOI: $10.3892 /$ ijmm.2018.3974

\begin{abstract}
The overall incidence rate of stroke is increasing worldwide. Inflammatory damage following a stroke is a leading cause for the poor prognosis and high disability rate observed in stroke patients. Microglia are considered to be the main causative agents of inflammatory injury following cerebral infarction, as they secrete various inflammatory cytokines and cytotoxic factors. The aim of the present study was to identify novel methods for attenuating inflammatory injury and improving the prognosis of stroke patients. Lipopolysaccharide-stimulated microglia were treated using propofol in vitro and a transient middle cerebral artery occlusion/reperfusion model was constructed in rats. Expression of cytotoxic factors, microglia proliferation and the neuroprotective effects of propofol were measured in vitro and in vivo. The in vitro studies demonstrated that propofol inhibits the expression of multiple cytotoxic factors, prevents structural changes to cytoskeletal proteins, and suppresses microglial migration via the adenosine $\mathrm{A} 2 \mathrm{~b}$ receptors. The results of the in vivo experiments revealed that propofol inhibits the abnormal proliferation of microglia, as well as reduces the expression levels of interleukin (IL)-6, IL-1 $\beta$, tumor necrosis factor $\alpha$, and the cytotoxic factor nitric oxide through the $\mathrm{A} 2 \mathrm{~b}$ receptor. In conclusion, propofol inhibited the excessive activation of microglia through the A2b receptor and attenuated the inflammatory injury following cerebral infarction. The current study may provide a reliable basis for further clinical studies on propofol and its putative
\end{abstract}

Correspondence to: Professor Mingming Dai, Department of Internal Neurology, The Second Affiliated Hospital, Hainan Medical University, 48 Baishui Pond Road, Longhua, Haikou, Hainan 570311, P.R. China

E-mail: 18580701104@163.com

Key words: cerebral infarction, microglia, inflammatory damage, propofol, adenosine A2b receptor role in improving the prognosis of patients with cerebral infarction.

\section{Introduction}

Ischemic cerebrovascular disease poses an increasing threat to human health due to its high rate of incidence and resulting disability (1). Rate of recurrence and mortality associated with ischemic cerebrovascular disease in Beijing, China was estimated as $27 \%$ (1). Cerebral ischemia/reperfusion injury is the leading cause of poor prognosis and high rate of severe disability observed in the clinical practice (2-4). The pathophysiology of this disease involves a variety of immune cells of the central nervous system, mainly microglia, which excessively activate and release a large quantity of oxygen free radicals, inflammatory cytokines and other pro-inflammatory compounds. The inflammatory injury then leads to neuronal death and increases the damage resulting from the stroke (5-7). Therefore, the focus of stroke research has become the identification of an effective drug that can suppress inflammation and improve patient prognosis.

Propofol is widely used in clinical practice as an intravenous anesthetic due to its rapid induction and recovery times (8). Recent research has identified that the mechanism of action of propofol in sedation may be associated with adenosine receptors $(9,10)$. Propofol inhibits adenosine reabsorption and increases the concentrations of extracellular adenosine (11). Adenosine receptors A1 and A2 are widely present in brain tissues. Subsequent to activation by adenosine, A1 and A2 receptors exert neuroprotective effects by increasing the intracellular cyclic adenosine monophosphate level, promoting glycogen decomposition, inhibiting the activation of central nervous system immune cells and improving the utilization rate of metabolic substrates (12-14).

Therefore, in the present study, it was hypothesized that propofol may activate the $\mathrm{A} 2 \mathrm{~b}$ receptor and inhibit microglial activation, thereby reducing inflammatory injury following cerebral infarction. Through a number of in vitro and in vivo experiments, alterations in the microglia activation conditions, the levels of cytotoxic molecules and the expression of inflammatory factors were detected following treatment with propofol. 


\section{Materials and methods}

Microglia isolation and culture. All procedures involving animals were reviewed and approved by the Institutional Clinical Experiments Committee and Institutional Review Board of the Hainan Medical University (Haikou, China). A total of 90 male Sprague-Dawley (SD) rats weighing 250-300 g were sacrificed by overdose of anesthetic at 3 days after birth. The microglia isolation procedure was performed as previously described $(15,16)$. Briefly, brains were removed and washed with sterile phosphate-buffered saline (PBS). Meninges and brain blood vessels were stripped, and the bilateral cerebral cortex was cut into sections and digested for $10 \mathrm{~min}$ in trypsin. Next, the cells were filtered (port size, $70 \mu \mathrm{m}$ ), seeded on poly-L-lysine-coated flasks at a density of $4 \times 10^{5} / \mathrm{ml}$ and cultured in Dulbecco's modified Eagle's medium (DMEM)-F12 medium (Invitrogen; Thermo Fisher Scientific, Inc., Waltham, MA, USA) containing $10 \%$ fetal bovine serum (FBS; Invitrogen; Thermo Fisher Scientific, Inc.) at $37^{\circ} \mathrm{C}$. After the cells had grown to cover the bottom of the culture flasks, the flasks were shaken using a rotatory shaker (Cellnest Shaker; Sino-Biotop, Shanghai, China) at a speed of $200 \mathrm{rpm}$ for $2 \mathrm{~h}$ at $37^{\circ} \mathrm{C}$. Subsequently, cells $\left(1 \times 10^{6} / \mathrm{ml}\right)$ were collected and seeded into $250 \mathrm{ml}$ flasks and 6-well culture plates. Non-adherent cells were washed away after $1 \mathrm{~h}$, and fresh medium was added into all the culture flasks and plates.

For in vitro experiments, five groups of microglia were stimulated for 24-48 h respectively with $1 \mu \mathrm{g} / \mathrm{ml}$ lipopolysaccharide (LPS; Sigma-Aldrich; Merck KGaA, Darmstadt, Germany; $\mathrm{n}=8$ ), with LPS and 1,3 or $5 \mu \mathrm{g} / \mathrm{ml}$ propofol (AstraZeneca plc, Cambridge, UK; $n=8$ ), or with LPS, $5 \mu \mathrm{g} / \mathrm{ml}$ propofol and $100 \mu \mathrm{M}$ MRS agar (Sigma-Aldrich; Merck KGaA) $(\mathrm{n}=8)$. The control group was only treated with DMEM/F12 medium $(\mathrm{n}=8)$.

Reactive oxygen species (ROS) and nitric oxide (NO) detection. For ROS detection, pretreated microglia in each group were incubated with the molecular probe 2',7'-dichlorodihydrofluorescein diacetate (DCFH-DA; $10 \mu \mathrm{M}$; Sigma-Aldrich; Merck KGaA) in serum-free medium at $37^{\circ} \mathrm{C}$ for $1 \mathrm{~h}$ in the dark and then washed twice with PBS $(17,18)$. ROS levels were measured at $492 / 520 \mathrm{~nm}$ using a microplate reader (Synergy HT; BioTek Instruments, Inc., Winooski, VT, USA).

The total NO levels in microglia were measured using the Griess reagent kit (Invitrogen; Thermo Fisher Scientific, Inc.). Pretreated microglia were incubated with Griess reagent in serum-free medium at $37^{\circ} \mathrm{C}$ for $20 \mathrm{~min}$ in the dark and then washed twice with PBS (19). The control group only contained complete medium and Griess reagent. NO levels were measured at $540 \mathrm{~nm}$ using the microplate reader.

Immunofluorescence staining of F-actin. Microglia from each test group were respectively harvested, seeded $\left(1 \times 10^{6} / \mathrm{ml}\right)$ and stimulated on glass cover slips in 12-well poly-L-lysine-coated culture plates at $37^{\circ} \mathrm{C}$. Following fixation and permeabilization, $3 \%$ goat serum (R\&D Systems, Inc., Minneapolis, MN, USA) was used to block non-specific microglial proteins for $0.5 \mathrm{~h}$ at room temperature. Subsequently,cells were stained with $5 \mu \mathrm{g} / \mathrm{ml}$ FITC-phalloidin (Invitrogen; Thermo Fisher Scientific, Inc.) at $24^{\circ} \mathrm{C}$ for $1 \mathrm{~h}$ and washed with PBS (20-22). Next, $100 \mathrm{ng} / \mathrm{ml}$
DAPI (Invitrogen; Thermo Fisher Scientific, Inc.) was added into the culture plates, and the nuclei were stained for $15 \mathrm{~min}$. Finally, cells were imaged using a confocal imaging system (Leica TCS SPE; Leica Microsystems GmbH, Wetzlar, Germany).

MTT assay. Microglia from each test group were seeded on glass coverslips in 96 -well culture plates $\left(1 \times 10^{4}\right.$ cells per well) at $37^{\circ} \mathrm{C}$. Next, $5 \%$ MTT solution $(0.2 \mathrm{mg} / \mathrm{ml}$; Sigma-Aldrich; Merck KGaA) was added into each well ( $20 \mu \mathrm{l}$ per well) on the following day. After $4 \mathrm{~h}$, dimethyl sulfoxide (D4540; Sigma-Aldrich; Merck KGaA) was added to each well (200 $\mu \mathrm{l}$ per well). Finally, a microplate reader was used to measure the absorbance of each well at $490 \mathrm{~nm}$.

In vitro migration assay. Microglia were harvested and seeded into the upper chamber of a 12 -well Transwell plate $(0.65 \mu \mathrm{m}$; Corning, Inc., Corning, NY, USA). The lower chamber contained $1 \mu \mathrm{g} / \mathrm{ml}$ LPS, LPS and $5 \mu \mathrm{g} / \mathrm{ml}$ propofol, or LPS with $5 \mu \mathrm{g} / \mathrm{ml}$ propofol and $100 \mu \mathrm{M}$ MRS agar. The control group only contained culture medium. After 12 -h incubation at $37^{\circ} \mathrm{C}$, cells on the lower chamber were fixed and stained with crystal violet. The number of migrating microglia was observed under a light microscope (Leica DVM6; Leica Microsystems $\mathrm{GmbH})$.

In vitro scratch wound assay. Microglia $\left(1 \times 10^{6} / \mathrm{ml}\right)$ were plated onto a 12-well tissue culture plate and allowed to reach near-confluence overnight at $4^{\circ} \mathrm{C}$. Mitomycin $(5 \mu \mathrm{g} / \mathrm{ml}$; Bio-Rad Laboratories, Inc., Hercules, CA, USA) was added to the culture medium to inhibit cell proliferation at $2 \mathrm{~h}$ before the scratch wound was applied. Each well was scratched across the center using a sterile P-200 pipet tip to create an artificial in vitro wound. Cells in each group were treated as described earlier. After 12 -h incubation at $37^{\circ} \mathrm{C}$, the cells were washed with PBS and stained with $0.5 \%$ crystal violet. Imaging as performed by light microscopy and analyzed with ImageJ software (version 1.48; National Institutes of Health, Bethesda, MD, USA).

Animal transient middle cerebral artery occlusion (tMCAO) model. A total of 40 male SD rats (age, 8-12 weeks; weight, 220-250 g) were selected and purchased from the Experimental Animal Center of Hainan Medical University 5 days before the experiments. All procedures performed with animals were approved by the Institutional Clinical Experiments Committee of the Second Affiliated Hospital of Hainan Medical University (Haikou, China). Rats were housed at $21-26^{\circ} \mathrm{C}$ with a humidity of $65 \pm 5 \%, 0.03 \% \mathrm{CO}_{2}$ and $12 \mathrm{~h}$ light/dark cycle with free access to water and food. They were fasted for $6 \mathrm{~h}$ before tMCAO. Briefly, $2.5 \%$ sodium pentobarbital ( $36 \mathrm{mg} / \mathrm{kg}$; Sigma-Aldrich; Merck KGaA) was injected into the abdominal cavity of rats. Next, the common, internal and external carotid arteries of the neck were exposed by blunt dissection. A $1.8 \mathrm{~cm}$-long nylon filament (diameter, 0.24-0.28 mm; Biospes Co., Ltd., Chongqing, China) was inserted via a cut of the internal carotid artery, and the middle cerebral artery was occluded by inserting the nylon filament $(23,24)$. Rats were kept at $37^{\circ} \mathrm{C}$ during the entire surgical procedure. The nylon filament was untied $2 \mathrm{~h}$ after tMCAO. 
Finally, the rats in the experimental group were treated with propofol (100 mg/kg; Sigma-Aldrich; Merck KGaA), while the rats without any surgery in the sham group and rats subjected to tMCAO in control group were only treated with saline (100 mg/kg; Sigma-Aldrich; Merck KGaA) $(25,26)$. A total of 10 animals were used for each group.

BrdU immunofluorescence. At 2 days after tMCAO challenge, SD rats were intravenously treated with BrdU (50 mg/kg; Sigma-Aldrich; Merck KGaA) two times at 12-h intervals. Then, rats were euthanized using $2.5 \%$ sodium pentobarbital. Cryostat brain sections $(20 \mu \mathrm{m})$ from the frontal to the occipital poles were cut using a cryomicrotome (HM525 NX; Thermo Fisher Scientific, Inc., Waltham, MA, USA). The sections were then incubated with $\mathrm{HCl}(2 \mathrm{~N})$ at $37^{\circ} \mathrm{C}$ for $30 \mathrm{~min}$, and borate buffer was used to neutralize the $\mathrm{HCl}$ through three washes. Next, the sections were blocked with $1 \%$ FBS in $0.3 \%$ Triton $\mathrm{X}-100$ at $37^{\circ} \mathrm{C}$ for $10 \mathrm{~min}$ and stained using a BrdU cell proliferation assay kit (Cell Signaling Technology, Inc., Danvers, MA, USA) following the manufacturer's protocol. Microglia were labeled with a primary antibody against ionized calcium binding adaptor molecule 1 (Iba1; cat. no. 019-19741; 1:500 dilution; Wako Pure Chemical Industries, Ltd., Osaka, Japan) at $4^{\circ} \mathrm{C}$ overnight. In addition, 702- Rat IgG1 kappa antibody (cat. no. 67-4301-80; 1:500; Thermo Fisher Scientific, Inc.) was used as the control antibody. Subsequently, the cells were stained with an Alexa488-conjugated secondary antibody (cat. no. S11223; 1:1,000 dilution; Thermo Fisher Scientific, Inc.) after the unbound primary antibody was washed three times using PBS. Finally, cells were imaged using laser scanning confocal microscopy (TCS SP8 DLS; Leica Microsystems $\mathrm{GmbH}$ ). The number of double immune positive microglia in 10 different fields-of-view was analyzed using Image J software.

ELISA. At $12 \mathrm{~h}$ after tMCAO, the cerebrospinal fluid (CSF) of SD rats was extracted following a previously described method (27). Subsequently, the levels of interleukin (IL)-1 $\beta$, IL-6 and tumor necrosis factor- $\alpha$ (TNF- $\alpha$ ) in the CSF were measured using ELISA kits (cat. nos. EK0393, EK0412 and EK0526; Boster Biological Technology, Ltd., Wuhan, China) following the manufacturer's protocol.

Statistical analysis. Experimental values are reported as the mean \pm standard deviation, and the one-way analysis of variance followed by Student-Newman-Keuls post hoc test was used to evaluate statistically significant differences between groups. Differences were considered to be statistically significant when the P-value was $<0.05$. Statistical evaluations were performed with the SPSS version 13 software package (SPSS, Inc., Chicago, IL, USA).

\section{Results}

Propofol inhibits NO and ROS overexpression induced by LPS in microglia through the $A 2 b$ receptor. ROS and NO are the major cytotoxic factors secreted by overactive microglia (Fig. 1). The present study results revealed that the expression levels of ROS and NO in microglia were
0.58 and $2.52 \mu \mathrm{M}$ in untreated cells (Fig. 2). Following stimulation with LPS $(1 \mu \mathrm{g} / \mathrm{ml})$, the levels of ROS and NO increased by 379.3 and $491.6 \%$ (both $\mathrm{P}<0.01$ ), respectively. However, the ROS and NO levels decreased by 8.3 and $12.2 \%$ as compared with the LPS alone group when LPS-treated microglia were stimulated with propofol $(1 \mathrm{mg} / \mathrm{l})(\mathrm{P}<0.01)$. When the concentration of propofol was increased to $3 \mathrm{mg} / \mathrm{l}$, the ROS and NO levels in LPS-treated cells decreased by 31.6 and $39.5 \%$, respectively, and further decreased by 52.3 and $49.8 \%$ following treatment with $5 \mathrm{mg} / 1$ propofol $(\mathrm{P}<0.01)$. However, the effect of propofol $(5 \mathrm{mg} / \mathrm{l})$ on microglia was inhibited by $100 \mu \mathrm{M}$ MRS agar, an A2b receptor antagonist, and the levels of ROS and NO were increased by 169.5 and $172.4 \%(\mathrm{P}<0.05)$, respectively, as compared with those in the LPS and propofol (5 mg/l) group (Fig. 2A and B).

Propofol inhibits structural alterations in cytoskeletal protein $F$-actin in microglia through $A 2 b$ receptors. The actin cytoskeleton serves an important role in the morphological alteration of cells, and structural change in F-actin is one of the results of microglial activation (Fig. 1) (28). In the present study, the changes in F-actin were detected by fluorescence staining (Fig. 3A). The results revealed that the fluorescence intensity of F-actin in microglia was low in untreated control cells, whereas it significantly increased following the addition of LPS. The fluorescence intensity in microglia strongly decreased subsequent to treatment with propofol, which demonstrated that propofol inhibited the structural alterations in the cytoskeletal protein in microglia. However, the effect of propofol on microglia was suppressed by the addition of $100 \mu \mathrm{M}$ MRS agar (Fig. 3A). MTT results demonstrated that microglial survival rates were similar among all four experimental groups, as the OD value was $\sim 1.0$ and there was no significant difference between any of the groups (Fig. 3B).

Propofol inhibits LPS-induced abnormal migration of microglia via the $A 2 b$ receptor. Microglia migration increased abnormally following cerebral infarction (Figs. 1 and 4) (29). In untreated control cells, the number of migrating cells was 473 and the migration distance was $0.195 \mathrm{~mm}$ (Fig. 4). Subsequent to treatment with LPS, microglial migration was significantly increased, as the number of migrating cells and the migration distance had increased to 236.7 and $412.5 \%$ ( $\mathrm{P}<0.01)$, respectively. However, microglial migration decreased following treatment with propofol $(5 \mathrm{mg} / \mathrm{ml})$, as the number of migrating cells and the migration distance markedly decreased by 46.4 and $54.1 \%$ ( $\mathrm{P}<0.01)$, respectively. These results demonstrated that propofol inhibited microglial migration following cerebral ischemia. Similar to the aforementioned results of other experiments, the effect of propofol was inhibited by MRS agar treatment, as the number of migrating cells and the migration distance in the MRS agar-treated group increased to 137.6 and $196.4 \%(\mathrm{P}<0.05)$, respectively, compared with that of the LPS and propofol (5 mg/l) group (Fig. 4A and B).

Propofol inhibits abnormal proliferation of microglia in the tMCAO model through the A2b receptor. Abnormal proliferative potential of microglia is associated with brain ischemia and reperfusion (30). In order to assess the effect of propofol 


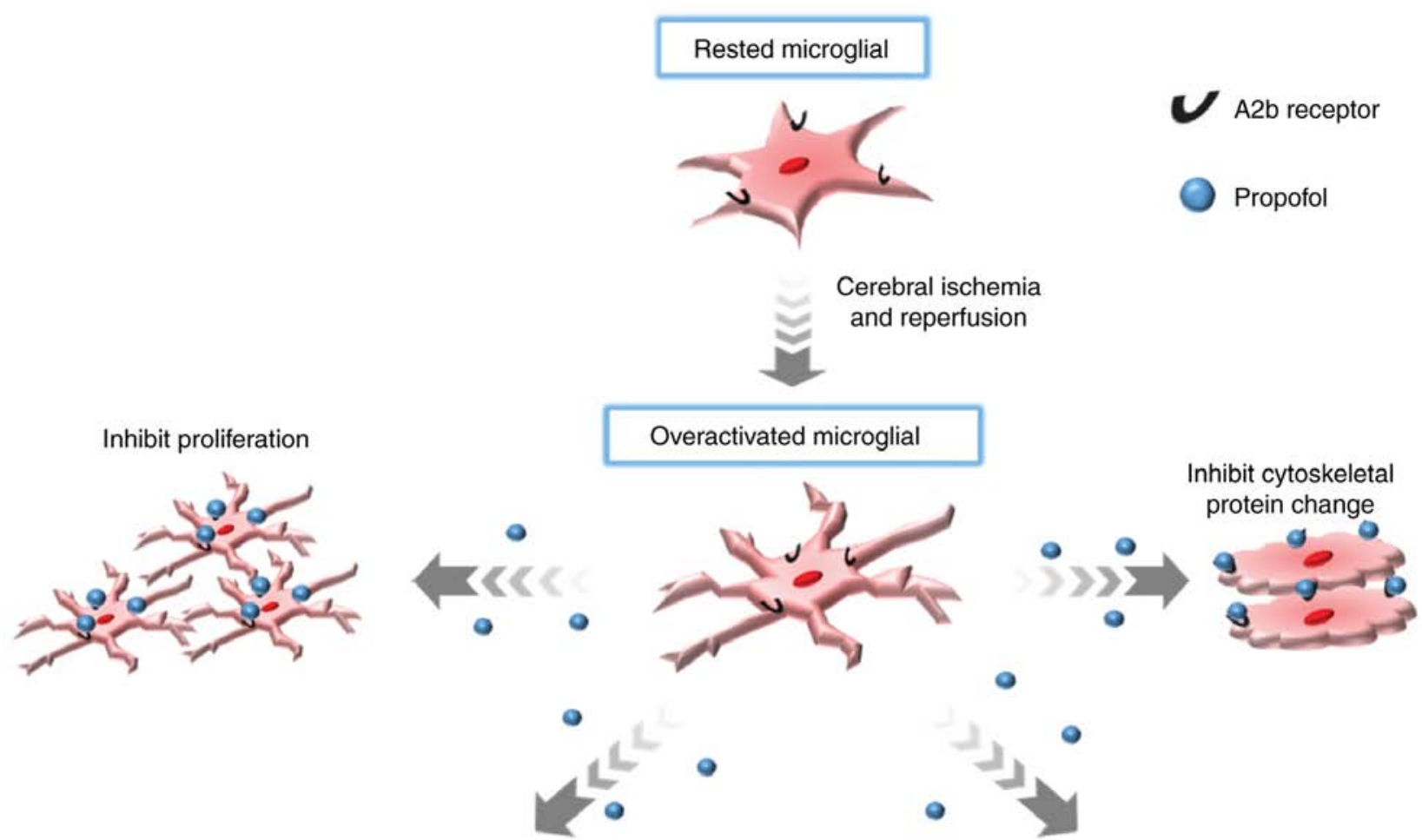

Inhibit migration

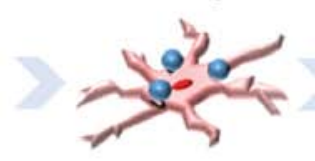

Inhibit expression of cytotoxic and inflammatory factors

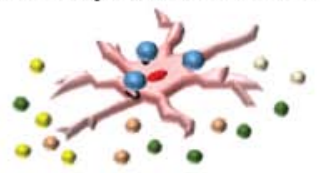

Figure 1. Graphical abstract of the effect of propofol on excessive activation of microglia. Microglia change from a resting state to an overactive state following cerebral ischemia and reperfusion through alterations in the cytoskeletal protein structure, proliferation, migration, and increased expression of inflammatory and cytotoxic compounds. Propofol inhibits these abnormal changes and the excessive activation of microglia through the adenosine A2b receptor, thereby attenuating inflammatory injury following cerebral infarction.
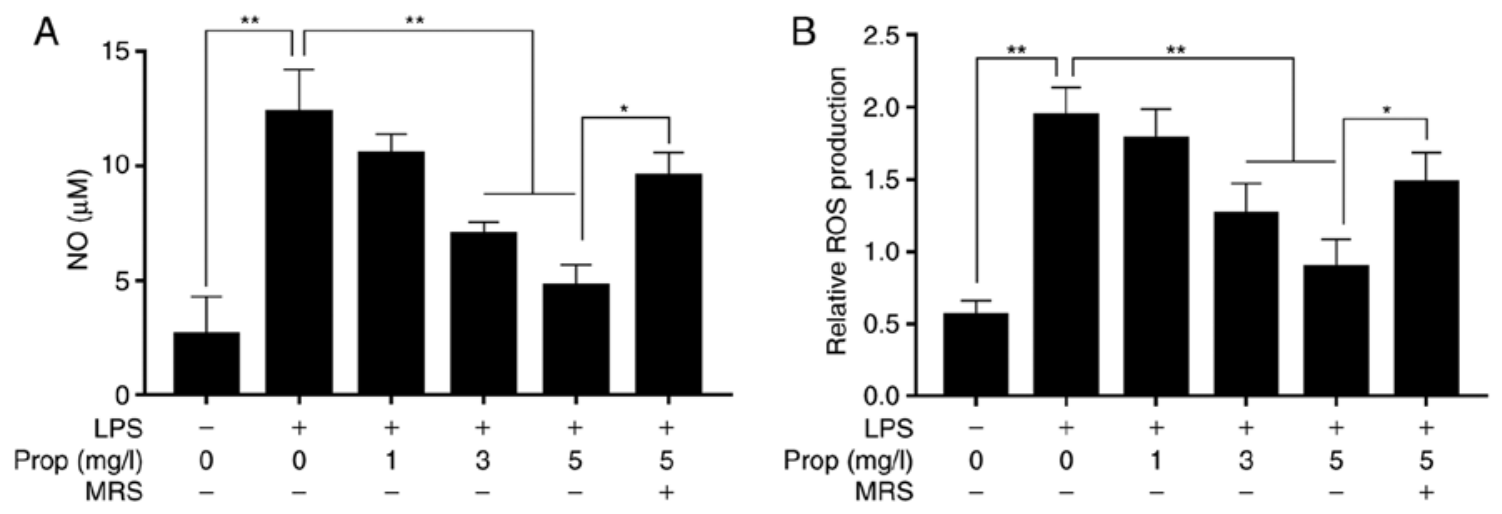

Figure 2. Expression levels of cytotoxicity factors were measured upon stimulation of LPS-treated microglia withpropofol (1,3,5 mg/l) or MRS agar (1 mg/l) for 2 days. (A) NO expression levels in microglia were quantified using Griess reagent kit. (B) ROS expression levels in microglia were quantified by an ROS assay kit. Values represent the mean \pm standard deviation ( $\mathrm{n}=8$ per group). ${ }^{*} \mathrm{P}<0.05$ and ${ }^{* * *} \mathrm{P}<0.01$. LPS, lipopolysaccharide; Prop, propofol; NO, nitric oxide; ROS, reactive oxygen species.

on microglial proliferation, brain sections were processed with double-immunofluorescence with BrdU and an antibody against Iba1, a microglia-specific marker. In the control group, microglia exhibited a low proliferative potential, and the number of double-positive cells was $28.6 / \mathrm{mm}^{2}$. Microglial proliferative potential increased significantly subsequent to
tMCAO, and the number of double-positive cells increased to $367.2 / \mathrm{mm}^{2}$ at 3 days following tMCAO $(\mathrm{P}<0.01)$. Upon treatment with propofol $(5 \mathrm{mg} / \mathrm{l})$, the number of double-positive cells in the tMCAO model rats decreased to $92 / \mathrm{mm}^{2}(\mathrm{P}<0.01)$. However, the effect of propofol was significantly inhibited by the addition of MRS agar, as the number of microglia 

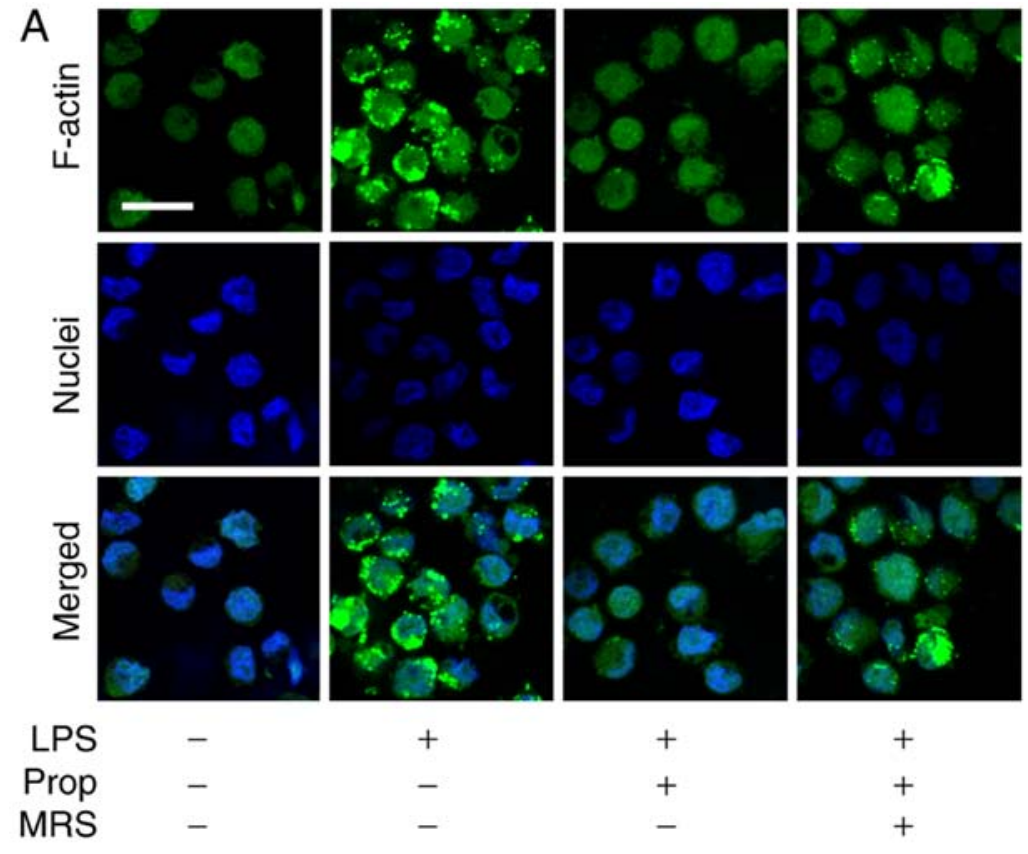
$+$

$+$

$+$

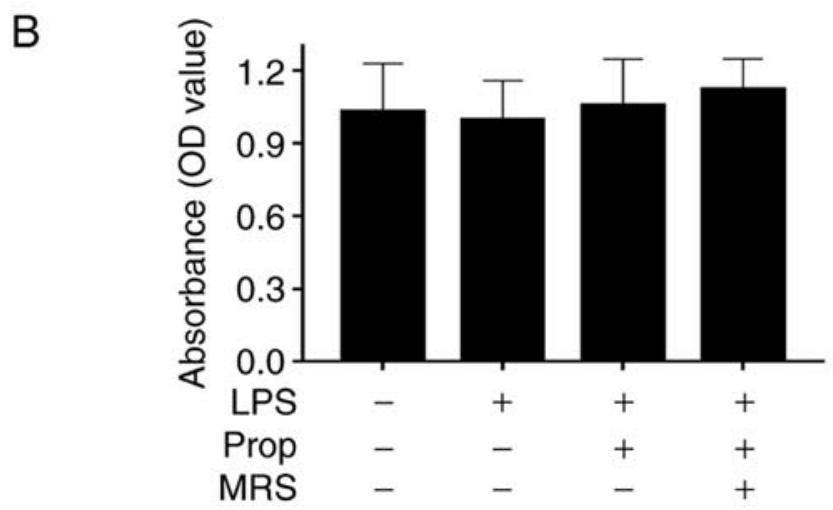

Figure 3. Structural alterations of F-actin in LPS-treated microgliastimulated with propofol $(5 \mathrm{mg} / \mathrm{l})$ or MRS agar (1 mg/l) for 2 days were assessed by immunofluorescence. (A) Representative images of immunofluorescence positive cells demonstrating the structure of F-actin in microglia. Microglial F-actin was stained with FITC-phalloidin (green), and cell nuclei were stained with DAPI (blue). Scale bar, $50 \mu \mathrm{m}$. (B) OD value assayed by Microplate Reader at $490 \mathrm{~nm}$ show the results of the MTT assay of microglial survival. $\mathrm{n}=8$ per group. Values represent the mean $\pm \mathrm{SD},{ }^{*} \mathrm{P}<0.05$ and ${ }^{* *} \mathrm{P}<0.01$. OD, optical density; LPS, lipopolysaccharide; Prop, propofol.

\section{A}
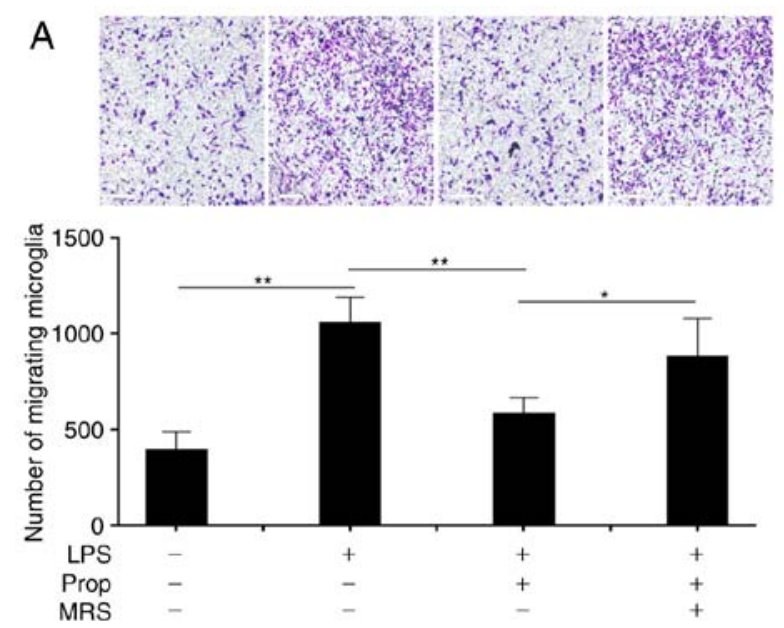

B
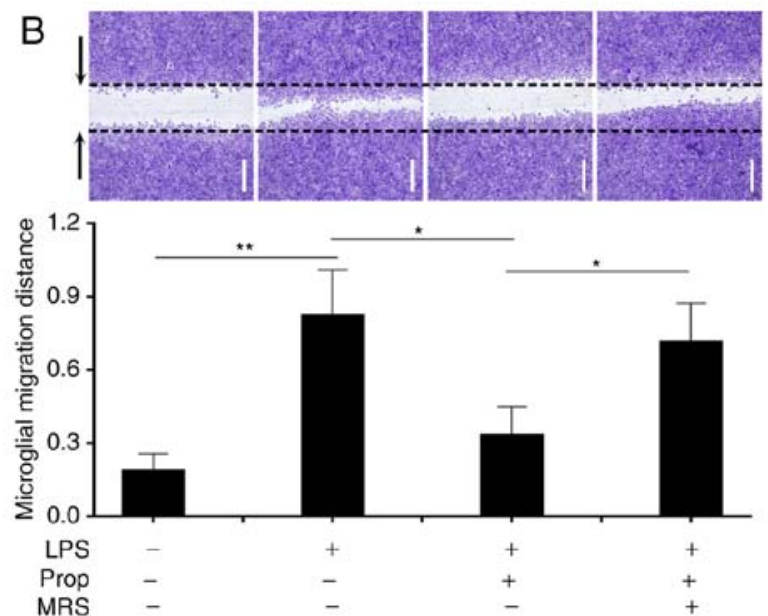

Figure 4. Effect of propofol on the abnormal migration of overactivated microglia. (A) Representative images and quantification of cells in the lower chamber demonstratedthe abnormal migration of microglia. In the Transwell assay, LPS with propofol (5 mg/l) or MRS agar (1 mg/l) was added to the lower chamber, and microglia were cultured in the upper chamber. (B) A scratch wound was created using a sterile P-200 pipette tip, and microglia were then treated using LPS with propofol or MRS agar for $12 \mathrm{~h}$. Representative images demonstrate the migration distance of microglia in the different groups. Values represent the mean \pm standard deviation ( $\mathrm{n}=8$ per group). ${ }^{*} \mathrm{P}<0.05$ and ${ }^{* *} \mathrm{P}<0.01$. LPS, lipopolysaccharide; Prop, propofol. 

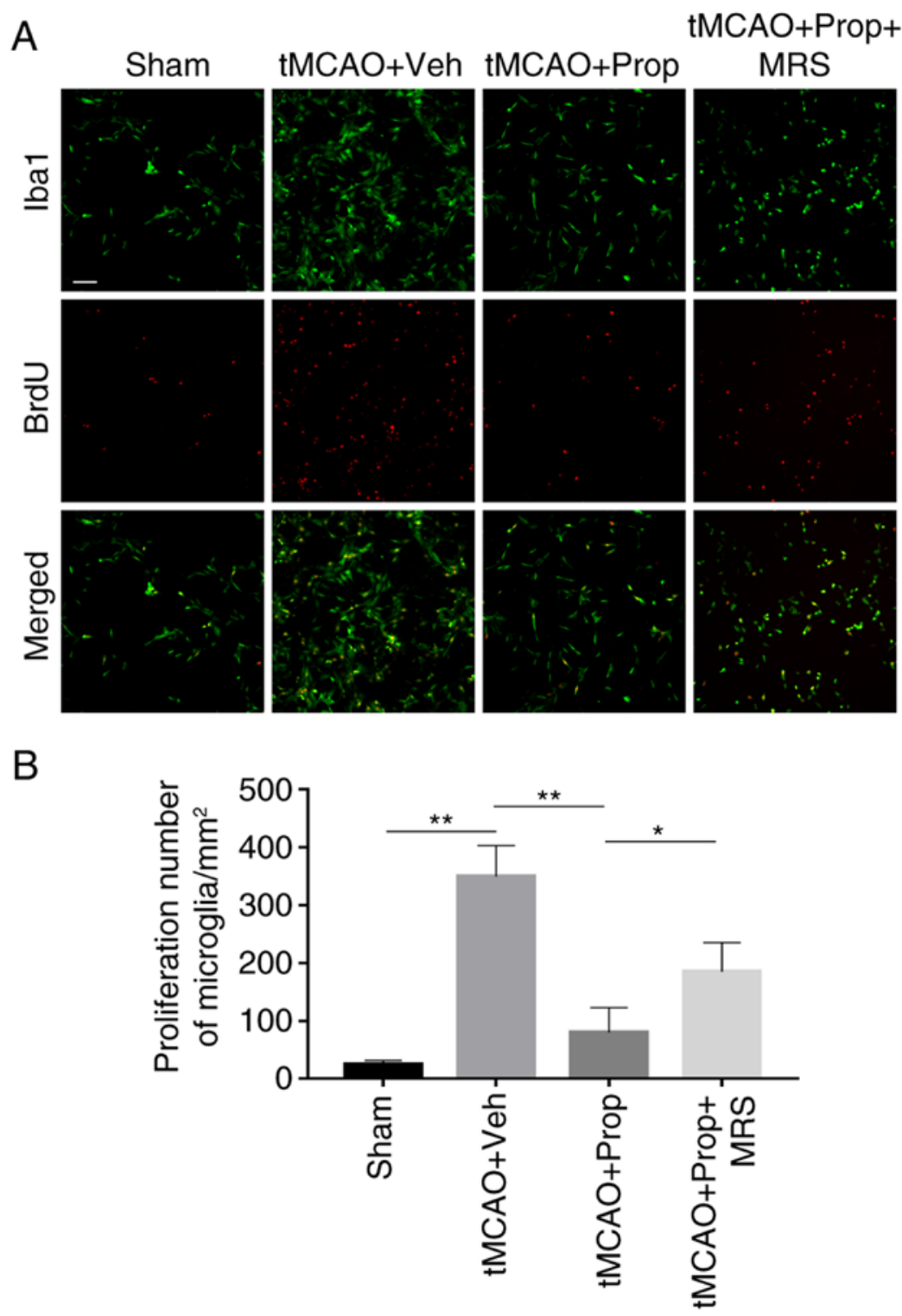

Figure 5. Proliferated microglia in the post-ischemic brain at 3 days after tMCAO. (A) Cryostat brain sections were obtained from rats and immunostained to observe the proliferated microglia at 3 days after tMCAO challenge in different groups. Iba1 (green) was used to stain microglia, and BrdU (red) was incorporated into proliferating cells in each group. Representative images demonstrated double-positive cells in the ischemic brain at 3 days after tMCAO. Scale bar, $50 \mu \mathrm{m}$. (B) The number of double-positive cells was counted to quantify the microglial proliferation at 3 days after tMCAO. Values represent the mean \pm standard deviation ( $\mathrm{n}=10$ per group). ${ }^{*} \mathrm{P}<0.05$ and ${ }^{* *} \mathrm{P}<0.01$. tMCAO, transient middle cerebral artery occlusion/reperfusion; Veh, vehicle; Prop, propofol.

increased to $196 / \mathrm{mm}^{2}$ in the MRS agar-treated group $(\mathrm{P}<0.05)$ (Fig. 5A and B).

Propofol inhibits the overexpression of microglial inflammatory factors in the $\mathrm{tMCAO}$ model via the A2b receptor. Following cerebral ischemia and reperfusion, microglia and other associated inflammatory cells release a large quantity of pro-inflammatory and cytotoxic factors, including IL-6, IL-1 $\beta$, TNF- $\alpha$ and NO. In the control group, the inflammatory response remained at a low level, and the measured levels of IL-6, IL-1 $\beta$ and TNF- $\alpha$ in the CSF were 461, 53 and $226 \mathrm{pg} / \mathrm{ml}$, respectively, while the level of NO was $0.0088 \mu \mathrm{mol} / \mathrm{mg}$. The inflammatory response was significantly enhanced at 3 days after tMCAO challenge, and the levels of IL-6, IL-1 $\beta$, TNF- $\alpha$ and NO increased to 294.1,
312.4, 255.7 and 283.4\%, respectively (all $\mathrm{P}<0.01$ ), compared with those observed in the control group. Following treatment with propofol $(5 \mathrm{mg} / \mathrm{ml})$, the inflammatory response decreased, and the production of IL-6, IL-1 $\beta$, TNF- $\alpha$ and NO decreased by $61(\mathrm{P}<0.05), 49(\mathrm{P}<0.01), 63(\mathrm{P}<0.05)$ and $47 \%(\mathrm{P}<0.01)$, respectively. However, the effect of propofol on microglia and other inflammatory cells was significantly inhibited by MRS agar treatment, as the production of IL-1 $\beta$, TNF- $\alpha$ and NO in the CSF increased by 1.33-, 1.41- and 1.39fold $(\mathrm{P}<0.05)$, respectively, when compared with the LPS and propofol-treated alone group. Notably, the expression of IL-6 was less affected by MRS agar treatment, and the IL-6 production only increased to 1.15 -fold that of the propofoltreated group in the MRS-treated group, with no significant difference observed (Fig. 6). 
A

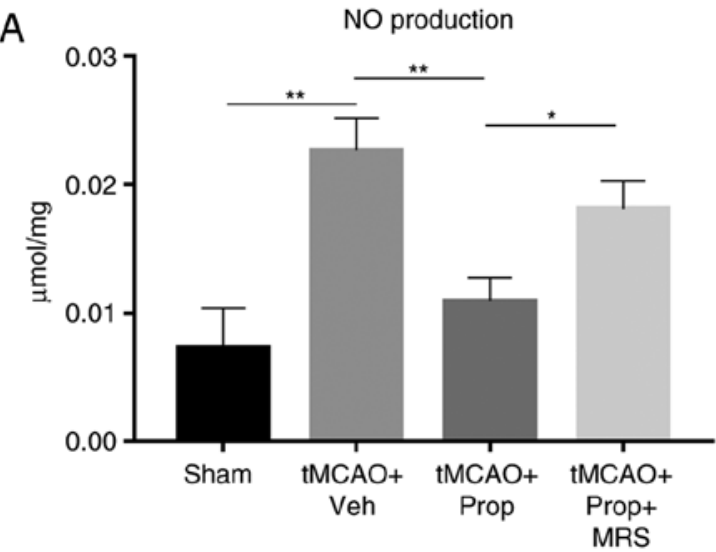

C

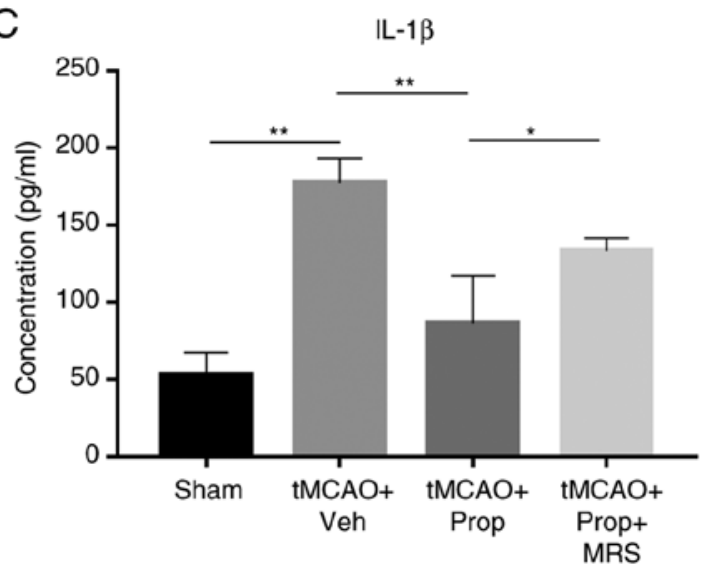

B
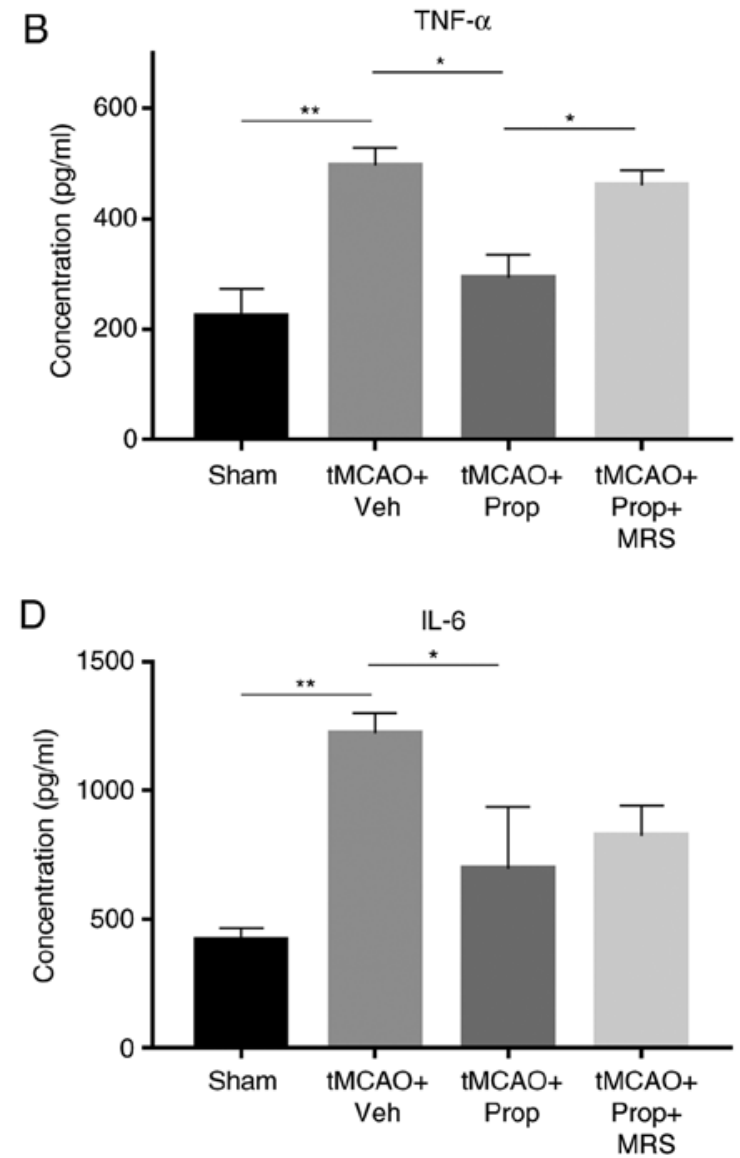

Figure 6. Expression levels of inflammatory factors in the post-ischemic brain. CSF was extracted from rats in the different groups at 3 days after tMCAO. The expression levels of (A) NO levels, (B) TNF- $\alpha$ and (C) IL-1 $\beta$ in the CSF were measured by ELISA, while (D) IL-6 levels were quantified using Greiss method. Values represent the mean \pm standard deviation ( $\mathrm{n}=10$ per group). ${ }^{*} \mathrm{P}<0.05$ and ${ }^{* * *} \mathrm{P}<0.01$. tMCAO, transient middle cerebral artery occlusion/reperfusion; CSF, cerebrospinal fluid; Veh, vehicle; Prop, propofol; NO, nitric oxide; TNF- $\alpha$, tumor necrosis factor $\alpha$; IL, interleukin.

\section{Discussion}

Excessive activation of microglia is considered to be the main cause of cerebral infarction injury in the central nervous system (31-32). Secreted cytotoxic and inflammatory cytokines can lead to neuritis, immune response and damage of the nervous system, and may be the main pathological basis of several neurodegenerative diseases (33). A number of previous studies had found that propofol has neuroprotective effects in animal models (34-36). However, there is a lack of studies on the mechanism of action of propofol regarding its neuroprotective role (37). In the present study, the effects of propofol on suppressing inflammation were examined, and it was attempted to identify a suitable dose for clinical use.

LPS can induce microglia to release abundant quantities of NO and ROS. These factors are major cytotoxic substances released following ischemic stroke $(38,39)$. Comparing microglia from each experimental group, the current study observed that propofol was able to inhibit the overexpression of NO and ROS, and these results are in agreement with recent evidence (40). In addition, the experimental results of the present study revealed that there was a negative correlation between the expression of cytotoxic factors and the propofol dose within a certain concentration range. Simultaneously, the effect of propofol on microglial activityand survival was also investigated by MTT assay, and the results indicated that propofol treatment has no significant effect on microglial activity. These findings are important for future clinical applications of propofol.

Following ischemic stroke, activated microglia migrates rapidly to the site of injury and mediates the inflammatory reaction (28). Comparing the number of migrated cells in the Trans well experiment and scratch injury assays conducted in the present study, it was observed that propofol inhibited the abnormal proliferation of overactive microglia. These in vitro experimental results preliminarily demonstrate that propofol is able to inhibit the aberrant migration of overactive microglia and the overexpression of cytotoxic factors.

It is known that cytoskeletal proteins serve an important role in maintaining cellular morphology and function (41). In the present study, fluorescence staining of microglial F-actin demonstrated that propofol treatment inhibited the structural alterations induced by LPS in the microglial cytoskeleton induced.

A tMCAO rat model was also employed in the current study to assess microglial proliferation and inflammatory factor secretion in vivo. It was observed that propofol inhibited the proliferation and activation of microglia. In addition, the levels of IL-1 $\beta$, IL- 6 , TNF- $\alpha$ and NO were evidently low in the propofol-treated group, which indicated that propofol attenuated inflammation in tMACO. Although it remains uncertain whether all these inflammatory factors are secreted only by microglia, it is likely that propofol 
inhibits the overall levels of inflammatory factors in the brain subsequent to ischemic stroke.

In the aforementioned experiments, an MRS agartreatment group was used to determine the mechanism of action of propofol on cerebral inflammation. The results suggested that MRS agar, an A $2 b$ receptor antagonist, effectively blocked nearly all the effects of propofol on microglia. This supports the hypothesis that the $\mathrm{A} 2 \mathrm{~b}$ receptor serves a significant role in the inflammation-attenuating effects of propofol. However, blocking the $\mathrm{A} 2 \mathrm{~b}$ receptor did not significantly affect the level of IL-6, suggesting that propofol may also fulfill its antiinflammatory function via another route.

In conclusion, propofol was demonstrated to inhibit excessive microglial activation, abnormal proliferation and migration, as well as the release of cytotoxic and inflammatory factors, and its effects were likely mediated by the $\mathrm{A} 2 \mathrm{~b}$ receptor. Therefore, the present study determined a feasible drug and an appropriate clinical dosage for the purpose of reducing inflammation and improving the prognosis in cerebral infarction. In addition, A2b receptor may be not the only receptor through which propofol acts on microglia, and other pathways remain to be explored (42). Determining the ideal reaction time, propofol dosage and possible side effects will be the direction of future research.

\section{Acknowledgements}

Not applicable.

\section{Funding}

This study was supported by the 2016 Cultivation Fund of Hainan Medical University (grant no. HY2016-07) and the Industry Research Project of Hainan Health and Family Planning Commission (grant no. 1601032054A2001).

\section{Availability of data and material}

The datasets used and/or analyzed during the current study are available from the corresponding author on reasonable request.

\section{Authors' contributions}

HY took part in all experiments and was the major contributor in writing the manuscript. XW analyzed and interpreted the data regarding cell experiments. FK and $\mathrm{ZC}$ established the tMCAO model and conducted other experiments in vivo. YM analyzed and interpreted data regarding experiments in vivo. All authors read and approved the final manuscript.

\section{Ethics approval and consent to participate}

All procedures involving animals were reviewed and approved by the Institutional Clinical Experiments Committee and Institutional Review Board of the Hainan Medical University (Haikou, China).

\section{Patient consent for publication}

Not applicable.

\section{Competing interests}

The authors declare that they have no competing interests.

\section{References}

1. Liu J,Zhao D, Wang W, Sun JY, Li Y and Jia YN: Trends regarding the incidence of recurrent stroke events in Beijing. Zhonghua Liu Xing Bing Xue Za Zhi 28: 437-440, 2007 (In Chinese).

2. Labeyrie C, Cauquil C, Sarov M, Adams D and Denier C: Cerebral infarction following subcutaneous immunoglobulin therapy for chronic inflammatory demyelinating polyradiculoneuropathy. Muscle Nerve 54: 166-167, 2016.

3. Numata K, Suzuki M, Mashiko R and Tokuda Y: Lethal bilateral cerebral infarction caused by moyamoya disease. OJM 109: 501, 2016.

4. Zhan R, Xu K, Pan J, Xu Q, Xu S and Shen J: Long noncoding RNA MEG3 mediated angiogenesis after cerebral infarction through regulating p53/X4 axis. Biochem Biophys Res Commun 490: 700-706, 2017.

5. Kwon SK, Ahn M, Song HJ, Kang SK, Jung SB, Harsha N, Jee S, Moon JY, Suh KS, Lee SD, et al: Nafamostat mesilate attenuates transient focal ischemia/reperfusion-induced brain injury via the inhibition of endoplasmic reticulum stress. Brain Res 1627: 12-20, 2015.

6. Yang S, Gao L, Lu F, Wang B, Gao F, Zhu G, Cai Z, Lai J and Yang $Q$ : Transcription factor myocyte enhancer factor $2 \mathrm{D}$ regulates interleukin-10 production in microglia to protect neuronal cells from inflammation-induced death. J Neuroinflammation 12: 33, 2015.

7. Brown A: Understanding the MIND phenotype: Macrophage/ microglia inflammation in neurocognitive disorders related to human immunodeficiency virus infection. Clin Transl Med 4: 7 , 2015.

8. Pessach I and Paret G: PICU propofol use, where do we go from here? Pediatr Crit Care Med 17: 273-275, 2016.

9. Shi SS, Yang WZ, Chen Y, Chen JP and Tu XK: Profol reduces inflammatory reaction and ischemic brain damage in cerebral ischemia in rats. Neurochem Res 39: 793-799, 2014.

10. Woldegerima N, Rosenblatt K and Mintz CD: Neurotoxic properties of propofol sedation following traumatic brain injury. Crit Care Med 44: 455-456, 2016.

11. Liu J, Gao XF, Ni W and Li JB: Effects of propofol on P2X7 receptors and the secretion of tumor necrosis factor- $\alpha$ in cultured astrocytes. Clin Exp Med 12: 31-37, 2012.

12. Perígolo-Vicente R, Ritt K, Gonçalves-de-Albuquerque CF, Castro-Faria-Neto HC, Paes-de-Carvalho R and Giestal-deAraujo E: IL-6, A1 and A2aR: A crosstalk that modulates BDNF and induces neuroprotection. Biochem Biophys Res Commun 449: 477-482, 2014.

13. Zywert A, Szkudelska K and Szkudelski T: Effects of adenosine $A_{1}$ receptor antagonism on insulin secretion from rat pancreatic islets. Physiol Res 60: 905-911, 2011.

14. Ohnishi M, Urasaki T, Ochiai H, Matsuoka K, Takeo S, Harada T, Ohsugi Y and Inoue A: Selective enhancement of wnt4 expression by cyclic AMP-associated cooperation between rat central astrocytes and microglia. Biochem Biophys Res Commun 467: 367-372, 2015.

15. Kim JB, Yu YM, Kim SW and Lee JK: Anti-inflammatory mechanism is involved in ethyl pyruvate-mediated efficacious neuroprotection in the postischemic brain. Brain Res 1060: 188-192, 2005.

16. Wang AL, Yu AC, He QH, Zhu X and Tso MO: AGEs mediated expression and secretion of TNF $\alpha$ in rat retinal microglia. Exp Eye Res 84: 905-913, 2007.

17. Guo H, Hu LM, Wang SX, Wang YL, Shi F, Li H, Liu Y, Kang LY and Gao XM: Neuroprotective effects of scutellarin against hypoxic-ischemic-induced cerebral injury via augmentation of antioxidant defense capacity. Chin J Physiol 54: 399-405, 2011.

18. Hands S, Sajjad MU, Newton MJ and Wyttenbach A: In vitro and in vivo aggregation of a fragment of huntingtin protein directly causes free radical production. J Biol Chem 286: 44512-44520, 2011.

19. Lee JK, Chung J, McAlpine FE and Tansey MG: Regulator of G-protein signaling-10 negatively regulates NF- $\kappa \mathrm{B}$ in microglia and neuroprotects dopaminergic neurons in hemiparkinsonian rats. J Neurosci 31: 11879-11888, 2011. 
20. Shen K, Tolbert CE, Guilluy C, Swaminathan VS Berginski ME, Burridge K, Superfine R and Campbell SL: The vinculin $\mathrm{C}$-terminal hairpin mediates $\mathrm{F}$-actin bundle formation, focal adhesion, and cell mechanical properties. J Biol Chem 286: 45103-45115, 2011.

21. Gorbatyuk V, Nguyen K, Podolnikova NP, Deshmukh L, Lin X, Ugarova TP and Vinogradova O: Skelemin association with $\alpha_{\mathrm{IIb}} \beta_{3}$ integrin: A structural mode. Biochemistry 53: 6766-6775, 2014.

22. Li X, Liu Y and Haas TA: Skelemin in integrin $\alpha_{\mathrm{IIb}} \beta_{3}$ mediated cell spreading. Biochemistry 52: 681-689, 2013.

23. Zuhayra M, Zhao Y, von Forstner C, Henze E, Gohlke P, Culman J and Lützen U: Activation of cerebral peroxisome proliferatoractivated receptors $\gamma(\operatorname{PPAR} \gamma)$ reduces neuronal damage in the substantia nigra after transient focal cerebral ischaemia in the rat. Neuropathol Appl Neurobiol 37: 738-752, 2011.

24. Cao LJ, Wang J, Hao PP, Sun CL and Chen YG: Effects of ulinastatin, a urinary trypsin inhibitor, on synaptic plasticity and spatial memory in a rat model of cerebral ischemia/reperfusion injury. Chin J Physiol 54: 435-442, 2011.

25. Stekiel TA, Conteny SJ, Roman RJ, Weber CA, Stadnicka A, Bosnjak ZJ, Greene AS and Moreno C: Pharmacogenomic strain differences in cardiovascular sensitivity to propofol. Anesthesiology 115: 1192-1200, 2011.

26. Tanaka K, Tsutsumi YM, Kinoshita M, Kakuta N, Hirose K, Kimura M and Oshita S: Differential effects of propofol and isoflurane on glucose utilization and insulin secretion. Life Sci 88: 96-103, 2011.

27. Mariappan TT, Kurawattimath V, Gautam SS, Kulkarni CP Kallem R, Taskar KS, Marathe PH and Mandlekar S: Estimation of the unbound brain concentration of P-glycoprotein substrates or nonsubstrates by a serial cerebrospinal fluid sampling technique in rats. Mol Pharm 11: 477-485, 2014.

28. Wessler S, Gimona M and Rieder G: Regulation of the actin cytoskeleton in Helicobacter pylori-induced migration and invasive growth of gastric epithelial cells. Cell Commun Signal 9: 27, 2011.

29. Davalos D, Grutzendler J, Yang G, Kim JV, Zuo Y, Jung S, Littman DR, Dustin ML and Gan WB: ATP mediates rapid microglial response to local brain injury in vivo. Nat Neurosci 8: $752-758,2005$

30. Campanella M, Sciorati C, Tarozzo G and Beltramo M: Flow cytometric analysis of inflammatory cells in ischemic rat brain Stroke 33: 586-592, 2002

31. Jin YL, Luo HL, Liu L, Cheng CF and Ho LC: Inhibitory effects of different concentrations of curcumin on excessive activation of microglia cultured in vitro. J Clin Rehabil Tissue Eng Res 15: 6951-6955, 2011
32. Stertz L, Magalhães PV and Kapczinski F: Is bipolar disorder an inflammatory condition? The relevance of microglia activation. Curr Opin Psychiatry 26: 19-26, 2013.

33. Kokubu Y, Yamaguchi $\mathrm{T}$ and Kawabata $\mathrm{K}$ : In vitro model of cerebral ischemia by using brain microvascular endothelial cells derived from human induced pluripotent stem cells. Biochem Biophys Res Commun 486: 577-583, 2017.

34. Zhou R, Yang Z, Tang X, Tan Y, Wu X and Liu F: Propofol protects against focal cerebral ischemia via inhibition of microglia-mediated proinflammatory cytokines in a rat model of experimental stroke. Plos One 8: e82729, 2013

35. Li J, Han B, Ma X and Qi S: The effects of propofol on hippocampal caspase-3 and Bcl-2 expression following forebrain ischemia-reperfusion in rats. Brain Res 1356: 11-23, 2010.

36. Harman F, Hasturk AE, Yaman M, Arca T, Kilinc K, Sargon MF and Kaptanoglu E: Neuroprotective effects of propofol, thiopental, etomidate, and midazolam in fetal rat brain in ischemia-reperfusion model. Childs Nerv Syst 28: 1055-1062, 2012.

37. Pfeilschifter W, Czech-Zechmeister B, Sujak M, Mirceska A, Koch A, Rami A, Steinmetz H, Foerch C, Huwiler A and Pfeilschifter J: Activation of sphingosine kinase 2 is an endogenous protective mechanism in cerebral ischemia. Biochem Biophys Res Commun 413: 212-217, 2011

38. Shuhua X, Ziyou L, Ling Y, Fei W and Sun G: A role of fluofide on free radical generation and oxidative stress in BV-2 microglia cells. Mediators Inflamm 2012: 102954, 2012.

39. Brites D and Femandes A: Neuroinflammation and depression: Microglia activation, extracellular mierovesicles and microRNA dysregulation. Front Cell Neurosci 9: 476, 2015.

40. Zheng X, Huang H, Liu J, Li M, Liu M and Luo T: Propofol attenuates inflammatory response in LPS-activated microglia by regulating the miR-155/SOCS1 pathway. Inflammation 41: 11-19, 2018.

41. Hagiwara S, Iwaska H, Hasegawa A, Hidaka S, Uno A, Kaori U, Uchida $\mathrm{T}$ and Noguchi $\mathrm{T}$ : Continuous hemodiafiltration therapy ameliorates LPS-induced systemic inflammation in a rat model. J Surg Res 171: 791-796, 2011.

42. Tang J, Chen X, Tu W, Guo Y, Zhao Z, Xue Q, Lin C, Xiao J, Sun X, Tao T, et al: Propofol inhibits the activation of p38 through up-regulating the expression of annexin A1 to exert its anti-inflammation effect. PLoS One 6: e27890, 2011. 